\title{
A Search Space Reduction Strategy and a Mathematical Model for Multistage Transmission Expansion Planning with $N-1$ Security Constrains
}

\author{
Emivan F. da Silva • Mohsen Rahmani . \\ Marcos J. Rider
}

Received: 23 December 2013 / Revised: 5 September 2014 / Accepted: 12 September 2014 / Published online: 30 September 2014 (C) Brazilian Society for Automatics-SBA 2014

\begin{abstract}
This paper proposes a linear disjunctive model to solve a multistage transmission expansion planning problem (MTEP) considering $N-1$ security constraints. The use of a disjunctive linear model guarantees finding the optimum solution of the problems using existing classical optimization methods. For large-scale systems, when finding the optimum or even high-quality solutions of the MTEP problem is not possible in polynomial time, a search space reduction methodology (SSRM) is proposed. By using SSRM, it is possible to obtain very high-quality solutions or in most cases the optimum solution of the MTEP problem. The $N-1$ security constraint indicates that the transmission system must be expanded in such a way that, despite the outage of a system line (a pre-defined set of contingencies), the system continues to operate properly. The model was implemented using a modelling language for mathematical programming (AMPL) and solved using the CPLEX, which is a commercial solver. The IEEE 24-bus, Colombian 93-bus, and Bolivian 57-bus systems are used to evaluate and show the performance of the proposed mathematical model and the search space reduction strategy.
\end{abstract}

\section{E. F. da Silva}

Departamento de Matemática, Universidade do Estado de Mato

Grosso (UNEMAT), Sinop, Mato Grosso, Brazil

e-mail: emivan@unemat.br

\section{Rahmani}

Electrical and Computer Engineering Department,

Carnegie Mellon University, Pittsburgh, PA, USA

e-mail: rahmani@ieee.org

M. J. Rider $(\bowtie)$

Departamento de Engenharia Elétrica, Universidade Estadual Paulista "Júlio de Mesquita Filho" (UNESP), Ilha Solteira, São Paulo, Brazil

e-mail: mjrider@dee.feis.unesp.br
Keywords Transmission network expansion planning . $N-1$ security constraints - Disjunctive linear model . Search space reduction methodology

\section{List of symbols}

\section{Parameters and Variables}

$\alpha_{t} \quad$ Discount factor used to calculate the net present value of the investment at stage $t$

$c_{i j} \quad$ The investment cost of transmission lines in corridor $i j$

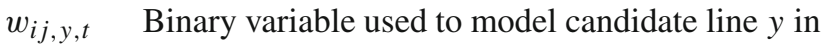
corridor $i j$, stage $t$

$f_{i j, y, c, t}$ Active power flow of candidate line $y$ in corridor $i j$, scenario $c$ and stage $t$

$f_{i j, c, t}^{0} \quad$ Active power flow of existing lines in the base topology in corridor $i j$, scenario $c$ and stage $t$

$g_{i, c, t} \quad$ Active power generation in bus $i$, contingency $c$ and stage $t$

$d_{i, t} \quad$ Active power demand in bus $i$ and stage $t$

$n_{i j}^{0} \quad$ Number of existing lines in the base topology in corridor $i j$

$N_{i j, c}^{\text {cont }}$ Elements of contingency matrix $N^{\text {cont }}$. For scenario $c$, it is equal 1 if an outage for a line in corridor $i j$ occurs; if equal 0 the line is in normal operation

$\theta_{i, c, t} \quad$ Voltage phase angle in bus $i$, scenario $c$ and stage $t$

$x_{i j} \quad$ Reactants of transmission line in corridor $i j$

$\bar{f}_{i j, c} \quad$ Maximum active power flow for a line in corridor $i j$ and scenario $c$

$M \quad$ Enough large number used in disjunctive model 


\section{$\bar{g}_{i, t, c} \quad$ Maximum active power generation in bus $i$, scenario $c$ and stage $t$ \\ $\bar{n}_{i j} \quad$ Maximum number of lines that can be added to the corridor $i j$ \\ ref Reference bus}

\section{Sets}

$\Omega_{b} \quad$ Set of buses

$C \quad$ Set of operation scenarios, where $C \in C^{0} \cup C^{1} \cup$ $C^{2}$ contains three different scenarios: the base case scenario (without contingency in lines) $C^{0}$, the set of contingency scenarios in existing lines $C^{1}$ and the set of contingency scenarios in candidate lines $C^{2}$. Note that each scenario $c \in C$ represents an operation state of the system

$T \quad$ Set of stages

$\Omega_{l} \quad$ Set of corridors

$Y \quad$ Set of candidate transmission lines

$E_{t} \quad$ Set of solutions in stage $t$

\section{Introduction}

The main objective of the multistage transmission expansion planning (MTEP) is to find the optimal expansion plan of a transmission system, that is, to specify circuits (transmission lines and/or transformers) that must be installed on the network in order to make its operation viable for a pre-defined planning horizon at minimum cost. The optimal expansion plan of a transmission system should define "where," "how many" and "when" new circuits should be installed (Garver 1970; Haffner et al. 2001; Romero et al. 2002).

The simplified mathematical models are generally used to represent the electric network. Generally the active power and/or voltage angles are considered in simplified models; the transportation model, the DC model, and hybrid linear model are some examples that usually employed for solving this problem. Considering the planning period, the problem can be classified as a one stage (static) problem, or the planning horizon can be divided in several planning stages (multistage planning problem) (Romero et al. 2002).

The MTEP problem with security constraints is traditionally solved in two phases (Monticelli et al. 1982; Seifu et al. 1989; Verma et al. 2010). In the first phase, the TEP problem without security constraints is solved, and, in the second phase, more new circuits are added into the system in order to make its operation viable in case of outages in any lines belonging to a predefined list of contingencies. The advantage of this strategy is that it finds an expansion plan with a relatively low computational effort. The main disadvantage is that the obtained plan is not optimal. Additionally, the obtained plan with security constraints is highly influenced by the expansion plan of the first phase, especially in large and complex systems (Silva et al. 2005).

In Oliveira et al. (2004), the MTEP with security constraints was solved considering multiple dispatch scenarios. A mixed integer nonlinear programming problem was proposed, and then a disjunctive formulation was used to linearise the nonlinear constraints.

Finally the authors used a heuristic method to find a solution for the MTEP with security based on the solutions of each stages.

A specialized genetic algorithm (SGA) was used in Silva et al. (2005) to solve the TEP with security constraints. A mixed integer nonlinear programming formulation was employed for modelling the problem. A static multi-objective TEP problem considering security constraints in the electricity market was proposed in Maghouli et al. (2009). They used an SGA, based on the NSGA II, together with a fuzzy decision analysis to solve the problem.

Lu et al. (2005) used a genetic algorithm to minimize the investment cost and the expected energy not supplied (EENS) in a competitive electricity market. They used the EENS technique to ensure the reliability and security of the system. Samarakoon et al. (2001), Xu et al. (2006, 2009), Asadamongkol and Eua-arporn (2009), Verma et al. (2009) proposed different heuristic algorithms for solving the transmission expansion planning problem considering $N-1$ security constraints.

A disjunctive linear model for MTEP problem considering the $N-1$ security constraints is proposed in this paper. The advantage of this model is that it can be solved using a commercial solver, without using heuristic algorithms. As the model is mixed integer linear, the optimum solution of the problem can be obtained, if given enough time. However, when the size of the systems increases, the time required for solving the problem increases exponentially, which makes the mixed integer problem intractable by the solver. In order to solve MTEP with $N-1$ security constraints for large-scale systems, this paper proposes a novel search space reduction methodology (SSRM) to reduce the combinatorial search space of the problem, which results in less computational time. Although the access to the optimum solution may not be guaranteed using this methodology, very high-quality solutions can be obtained. This is justified by comparing the optimum solution of the problems, obtained in full space, with the solution obtained with SSRM approach.

The article (da Silva et al. 2013) presents a similar study. But uses a very simple and an inefficient search space reduction strategy. The results are preliminary and the case studies are limited. However, the article presented here has more reliable results and the analyses are more rigorous.

The paper (Rahmani et al. 2013) uses a different strategy to reduce the search space, and they use forward and backward solution strategies; contingency or security is not 
addressed in this article that makes it even more different and less efficient.

The $N-1$ security criterion ensures that the transmission system must be expanded in such a way that despite a line outage it should still operate properly. The pre-defined set of contingencies can contain both existing lines in the base topology and the candidate lines. The IEEE 24-buses, Colombian 93-buses, and Bolivian 57-buses systems are used to show the efficiency of the proposed model. In Colombian and Bolivian systems, the search space reduction methodology (SSRM) is used to reduce the computational effort of the proposed model.

The paper is organized as follows: Sect. 2 presents the mathematical modelling of the problem. In Sect. 3, the proposed search space reduction strategy (SSRM) is presented. In Sect. 4, we present a brief description of the test systems, and then the obtained results are discussed for both planning with and without contingencies. Finally, Sect. 5 presents the conclusions.

\section{Mathematical Modelling}

The disjunctive linear model for multistage TEP problem with $N-1$ security constraints is presented in this section. In order to model $N-1$ security constraints in the MTEP problem, the contingency matrix $N^{\text {cont }}$ is introduced. The size of this matrix is $\left|\Omega_{l}\right| \times|C|$, and it composes of 0 and 1 elements. when $N_{i j, c}^{\text {cont }}=1$ indicates that $N-1$ contingency has occurred in corridor $i j$ in scenario $c$; otherwise $N_{i j, c}^{\text {cont }}=0$. Note that the continuous or operation variables, $f_{i j, y, c, t}, g_{i, c, t}, \theta_{i, c, t}$, as well as network constraints depend on the scenario $c$. Therefore, the number of operation variables increases $|C|$ times, this means that the number of operation variables linearly increases with respect to the number of contingencies $|C|$. However, since the investment variables are not dependent to the contingencies scenarios, the number of binary variables $\left(w_{i j, y, t}\right)$ is equal to the multistage planning without contingencies.

$$
\begin{aligned}
& \min v=\alpha_{1} \sum_{i j \in \Omega_{l}} \sum_{y \in Y} c_{i j} w_{i j, y, 1} \\
& +\sum_{t \in T, t>1} \alpha_{t} \sum_{i j \in \Omega_{l}} \sum_{y \in Y} c_{i j}\left(w_{i j, y, t}-w_{i j, y, t-1}\right) \\
& \text { S.a. } \\
& \sum_{j i \in \Omega_{l}}\left(\sum_{y \in Y} f_{j i, y, c, t}+f_{j i, c, t}^{0}\right) \\
& -\sum_{i j \in \Omega_{l}}\left(\sum_{y \in Y} f_{i j, y, c, t}+f_{i j, c, t}^{0}\right)+g_{i, c, t}=d_{i, t} \\
& \forall i \in \Omega_{b}, \forall c \in C, \forall t \in T \\
& f_{i j, c, t}^{0}=\left(n_{i j}^{0}-N_{i j, c}^{\mathrm{cont}}\right) \frac{\left(\theta_{i, c, t}-\theta_{j, c, t}\right)}{x_{i j}}
\end{aligned}
$$

$$
\begin{gathered}
\forall i j \in \Omega_{l}, \forall c \in C, \forall t \in T \\
\left|f_{i j, c, t}^{0}\right| \leq\left(n_{i j}^{0}-N_{i j, c}^{\text {cont }}\right) \bar{f}_{i j, c} \\
\forall i j \in \Omega_{l}, \forall c \in C, \forall t \in T \\
\left|x_{i j} f_{i j, y, c, t}-\left(\theta_{i, c, t}-\theta_{j, c, t}\right)\right| \leq M\left(1-w_{i j, y, t}\right) \\
\forall i j \in \Omega_{l}, \forall y \in Y, \forall c \in C \mid y>1, \forall t \in T \\
\left|x_{i j} f_{i j, y, c, t}-\left(\theta_{i, c, t}-\theta_{j, c, t}\right)\right| \leq M\left(1-w_{i j, y, t}\left(1-N_{i j, c}^{\text {cont }}\right)\right) \\
\forall i j \in \Omega_{l}, \forall y \in Y, \forall c \in C^{2} \mid y=1, \forall t \in T \\
\left|f_{i j, y, c, t}\right| \leq w_{i j, y, t} \bar{f}_{i j, c} \\
\forall i j \in \Omega_{l}, \forall y \in Y, \forall c \in C \mid y>1, \forall t \in T \\
\left|f_{i j, y, c, t}\right| \leq w_{i j, y, t} \bar{f}_{i j, c}\left(1-N_{i j, c}^{\text {cont }}\right) \\
\forall i j \in \Omega_{l}, \forall y \in Y, \forall c \in C{ }^{2} \mid y=1, \forall t \in T \\
0 \leq g_{i, c, t} \leq \bar{g}_{i, t, c} \forall i \in \Omega_{b}, \forall c \in C, \forall t \in T \\
\sum_{y \in Y} w_{i j, y, t} \leq \bar{n}_{i j} \quad \forall i j \in \Omega_{l}, \forall t \in T \\
w_{i j, y, t} \leq w_{i j, y-1, t} \\
\forall i j \in \Omega_{l}, \forall y \in Y / y>1, \forall t \in T \\
\theta_{i, c, t}=0 \quad \forall i \in \Omega_{b}, \forall c \in C / i=\operatorname{ref}, \forall t \in T \\
w_{i j, y, t} \text { binário } \forall i j \in \Omega_{l}, \forall y \in Y, \forall t \in T \\
w_{i j, y, t-1} \leq w_{i j, y, t} \\
\forall i j \in \Omega_{l}, \forall y \in Y, \forall t \in T / t>1
\end{gathered}
$$

In this model, the value of $M$ is calculated as reported in Vinasco et al. (2011). The binary variable $w_{i j, y, t}$ represents a line that can be (or not) added to a corridor. That is, $w_{i j, y, t}=1$ if the line $y$ is added to the corridor $i j$ and stage $t$; otherwise $w_{i j, y, t}=0$. Note that, if line $y$ is added to the corridor $i j$ in stage $t$, it should be presented in the subsequent stages, this condition is represented by constraint (12). Additionally, since in the proposed model each line is modelled separately, the active power flow $f_{i j, y, c, t}$ should also be provided separately for each line $y$ of corridor $i j$, scenario $c$ and stage $t$. For the base case $\left(c \in C^{0}\right)$, the maximum power flow for the corridor $i j$ is $\bar{f}_{i j, c}$ and the maximum generation is $\bar{g}_{i, t, c}$ in the bus $i$. But the maximum flow $\left(\bar{f}_{i j, c}\right)$ of line $y$ in contingency case $c \in C^{1} \cup C^{2}$ is assumed to be $10-20 \%$ greater than the normal value of the system. This assumption is usually considered for long-term transmission expansion planing (CEIDS 2012).

Objective function (1) represents the total investment cost of new transmission lines that will be added to the system. Constraint (2) is the power balance equation at bus $i$, scenario $c$ and stage $t$. Constraints (3) and (4) calculate the active power flow of existing lines in corridor $i j$ for scenario $c$ and stage $t$. Note that, if a contingency happens in existing transmission lines, which means $N_{i j, c}^{\text {cont }}=1$, an existing line is removed from the model, or, to express it more precisely, $n_{i j}^{0}$ is replaced by $\left(n_{i j}^{0}-1\right)$. 
Constraints (5), (6), (7), and (8) represent the active power flow expressions and limitations of candidate lines in scenario $c$ and stage $t$. Note that if $N_{i j, c}^{\text {cont }}=1$ in constraints (6) and (8), the first candidate line of the corridor $i j$ is removed from the system. Constraint (9) represents the generator active power limit for scenario $c$ in stage $t$. Constraint (10) is the new transmission lines limit that can be installed in corridor $i j$ in stage $t$. Constraint (11) guarantees the sequential installation of candidate lines in stage $t$. This constraint avoids similar solutions and results in fast convergence of the optimization problem. Constraint (12) defines that, if line $y$ is constructed in corridor $i j$ in stage $t-1$, it continues to operate in the next stages. This means that, if $w_{i j, y, t-1}=1 \Rightarrow w_{i j, y, t}=1$, and for this reason we need to calculate the investment of this line just once; so in the objective function (1), we have $\left(\sum_{y \in Y}\left(w_{i j, y, t}-w_{i j, y, t-1}\right)\right)$ to calculate the cost of new transmission lines, installed in the current stage and not in the previous stages. It is obvious that this rule applies for $t>1$. The binary feature of investment variables $w_{i j, y, t}$ is defined by (13). Constraint (14) requires that the phase angle of the reference bus $i$ becomes equal to zero in all of the scenarios and stages.

\section{Search Space Reduction Strategy}

As discussed in Sect. 2, we used a linear disjunctive model with some modifications to model the contingencies in transmission lines. The mixed integer linear formulation allows us to solve the problem using commercial solvers and obtain the optimum solution of the problem. But solving the proposed model in the full search space requires a great computational effort because of the numerous numbers of combinations that exist in the search space. Higher number of candidate lines, stages, and contingencies result in a higher number of combinations and, therefore, an enormous search space and computational effort. In cases of larger and more complex systems such as Colombian system, the problem is computationally intractable and the access to the optimum solution is impossible.

For this reason, we propose a heuristic algorithm that reduces the search space of the problem. This method, which creates a new and smaller search space, is called the search space reduction methodology (SSRM). In this method, the idea is to reduce the search space without changing the quality of the solution. The SSRM heuristic determines which solutions must remain in the search space and removes other unimportant solutions.

This section presents a new strategy to reduce combinatorial search space of the model presented in (1-14). The goal is to reduce the computational effort of solving largescale MTEP with $N-1$ security constraints and facilitate the convergence of commercial solver CPLEX. However, in SSRM, there is no guarantee that one obtains the optimal solution; nevertheless, a very high-quality solution and, in most cases, the optimal solution can be obtained for largescale systems.

In Vinasco et al. (2011), a heuristic algorithm is used to reduce the search space of the problem. But, the strategy of reducing the search space in here is different, because instead of finding a unique solution for each stage, $m$ solutions are found that guarantees a larger solution space with high probability of finding optimum solution.

It is very important to note that in TEP problems the number of lines, considered for installation (the solution), is much less than the number of candidate lines. In SSRM, the idea is to identify the prospective lines using a very fast method, or in another word to identify the lines that can significantly improve system performance or remove system bottlenecks. This fact is highlighted in multistage transmission expansion planning where the final solution of the system contains only a few numbers of lines.

The use of CPLEX solver in SSRM method is important as it can generate several distinct solutions for each stage and enables us to remove candidate lines with insignificant effects.

In this paper, the search space of MTEP problem with security constraints is simply reduced by re-defining the maximum number of transmission lines $\left(\bar{n}_{i j}\right)$ for constraint $(10)$, through the so-called algorithm SSRM.

The SSRM generates a reduced search space for the MTEP problem based on the solutions of a static TEP problem. The static TEP for stage $t$ can be solved using model (1-14), ignoring other stages and removing constraint (12). Considering $T$ as the set of planning stages, the CPLEX solver is used to generate a set of solutions ("pool") for each stage. The proposed strategy for finding a reduced combinatorial search space is provided in Fig. 1 and has the following steps:

1. Find a set $E_{t}$ with $m$ distinct solutions for each stage $t \in T$; each solution defines the number of transmission lines that can be installed in each corridor. Note that, as shown in Fig. 1, redundant solutions are removed from the set $E_{t}$.

2. Determine set $E=\cup_{t \in T} E_{t}$. The set $E$ contains all the solutions produced in step 1 .

3. The maximum value of the transmission lines in each corridor in the solutions set $E$ is considered as the upper bound value for the number of candidate transmission lines.

The $m$ distinct solutions result in a diverse solution set $E$, and consequently, this diverse search space guarantees high-quality solutions. To obtain $E_{t}, \forall t \in T$, in item (1), CPLEX solver directives are used to generate, manage and 


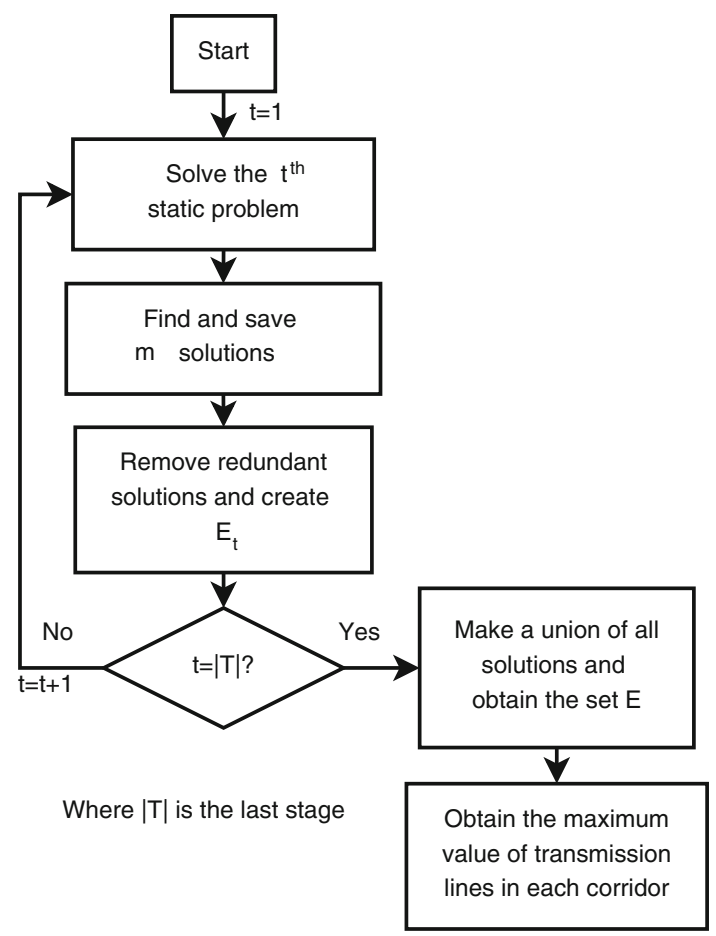

Fig. 1 The SSRM approach for reducing search space of the MTEP problem

store the solutions "pool" for the mixed integer programming problem. The poolstub directive is used to generate and save/retrieve the solutions (CPLEX 2010). The populatelim is used to limit the number of solutions added to the solution pool by the populate algorithm. Therefore, in SSRM methodology, we have poolstub = $\mathrm{m}$. The directive poolgap $=\mathrm{x}$ is used to find a solution with a predefined "gap" of $x \%$ with respect to the optimum solution.

The directives poolintensity $=\alpha$ and pool replace $=\beta$ of the CPLEX solver are used to diversify how $m$ solutions are selected. The value of $\alpha$ ranges from 0 to 4 , with 0 standing for a less intense search and 4 for a more intense search. If $\beta=0$, the selected solutions are the latest solutions added to the pool; if $\beta=1$, solutions are chosen according to the quality of objective function, and, if $\beta=2$, the solutions are selected randomly. Another directive that can be used is populate in order to generate more solutions; for more information see CPLEX (2010).

\section{Tests and Results}

The IEEE 24-bus system along with two real tests systems, Colombian 93-bus and Bolivian 57-bus systems, are used for the case studies. Both MTEPs with and without contingencies are solved for these systems. The solution of
MTEP problems for Colombian and Bolivian systems with security constraints cannot be obtained without using the SSRM. Also the literature does not yield report on the solution for these systems for MTEP problems with security constraints. Therefore, we were not able to compare all the results of this paper with other studies in the literature.

In order to study the MTEP considering the security constraints, a list of transmission lines with contingencies is required. One strategy is to identify those lines having most frequent outages from the historical data or based on the system operator experiences. Another strategy is to solve the proposed model without security constraints, and, from the solution, construct a contingency list based on the more congested lines (candidate or existing) (Wood et al. 2013).

The AMPL algebraic modelling language is employed to implement the proposed model (Fourer et al. 2003), and is solved using the CPLEX commercial solver (CPLEX 2010). The IEEE 24-bus, the Colombian 93-bus, and the Bolivian 57-bus systems are used to test and evaluate the mathematical model. The discount factors, to calculate the net present value of the investment cost, are chosen the same as the (Vinasco et al. 2011). For each system, two tests have been carried out: (a) MTEP without security constraints and (b) MTEP with $N-1$ security constraints. The results for the proposed model were obtained on a computer with $4 \mathrm{~Gb}$ RAM memory and Intel $® 8$ Core(TM) i7 processors.

\subsection{IEEE 24-Bus System}

The IEEE 24-bus system has 3 planning stages, 41 corridors, 10 generators, 17 load buses with a total amount of $8,550 \mathrm{MW}$ in the first stage, $8,988 \mathrm{MW}$ in the second stage and 9,437 MW in the third stage. A maximum of five lines can be constructed in each corridor. The IEEE 24-bus topology along with candidate lines is given in Fig. 2. The system's data and the discount factor for each stage can be found in LAPSEE (2012).

\subsubsection{IEEE 24-Bus System Without Security Constraints}

For the case without contingencies, the model has 3,536 constraints, 1,431 operation variables, and 615 binary variables. In the optimal solution, the lines $n_{6-10}=1, n_{7-8}=2$, $n_{10-12}=1$, and $n_{11-13}=1$ are considered for installation in the first stage, in the second stage $n_{20-23}=1$ is installed and in the third stage $n_{1-5}=1, n_{3-24}=1$ are installed. The total investment cost for this plan is about US\$220.28. This solution was found in $47.77 \mathrm{~s}$. This solution is also reported in Vinasco et al. (2011). Since the problem can be easily solved in full search space, we do not need to use the SSRM method in this case. The use of SSRM will be presented in more complicated problems. 


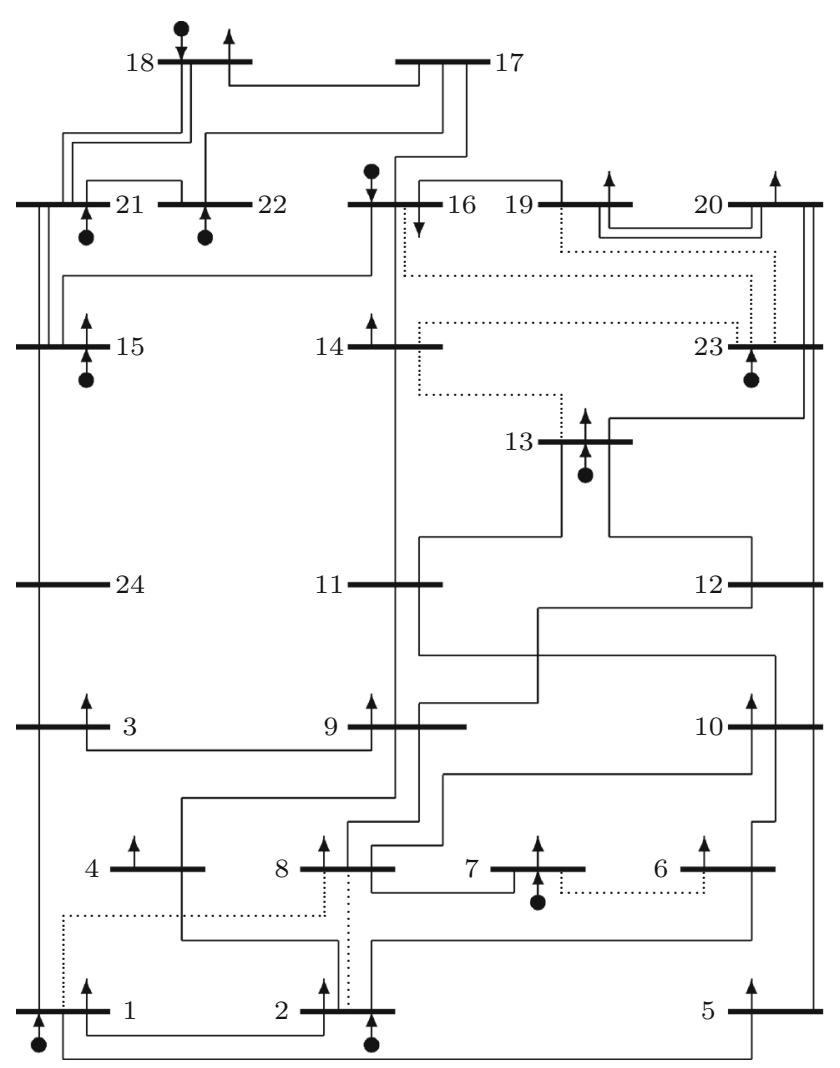

Fig. 2 The IEEE 24-bus system

\subsubsection{IEEE 24-Bus System with Security Constraints}

The contingency list of transmission lines prone to outage need to be defined prior to carrying out this test. The contingency list is obtained from the optimal plan of the case without contingency. Hence, the lines with a power flow greater than $80 \%$ of their maximum capacity are included in the contingency list. In this case, the contingency list is composed of 22 transmission lines $(1-5,2-4,3-24,6-10,7-8,9-11$, 9-12, 10-11, 10-12, 11-13, 12-13, 12-23, 14-16, 15-21, 15-24, 16-17, 17-18, 17-22, 18-21, 19-20, 20-23, 21-22), which represent approximately $53.65 \%$ of the total number of transmission lines.

Considering this contingency list, the mathematical model has 58,799 constraint, 28,516 operation variables, and 615 binary variables. The result of the test shows that the transmission lines $n_{1-5}=1, n_{3-24}=1, n_{4-9}=1, n_{6-10}=2$, $n_{7-8}=1, n_{10-12}=1, n_{15-24}=1$, and $n_{14-23}=1$ need to be constructed in the first stage, no lines installed for the second stage, and line $n_{7-8}=1$ is considered for the third stage. The optimum is found in 14,768.3 s and the investment cost for the optimal solution is equal to US\$ 362.64.

Again we do not need to use the SSRM method to reduce the search space of the problem, but we use that to show its excellent performance in reducing the search space of problem and lowering the computational time.
The following CPLEX directives are used: poolstub = solutions, populatelim $=5$, poolgap $=0.05$, poolintensity $=4$, poolreplace $=2$ and populate $=1$. In this case, the mathematical model has 32,022 constraints, 11,616 operation variables, and 315 binary variables. The solution obtained in this test is also optimal, but the processing time is $658.38 \mathrm{~s}$, which is much less than the processing time of the previous case, showing the efficiency of the proposed method. The tuning of the CPLEX directives is very important in solving the problems. Several tests, carried out by da Silva (2013), show the importance of using adequate values for the CPLEX directives.

\subsubsection{Comparison of the Results for IEEE 24-Bus System}

The optimal solution for the case without contingency needs eight new transmission lines for all three stages, while in the case with contingency, 10 transmission lines are needed. The investment cost of the planning with contingency is $64.63 \%$ greater than planning without contingency. Note that there are six common transmission lines, $\left(n_{6-10}=1, n_{7-8}=2\right.$, $\left.n_{10-12}=1, n_{1-5}=1, n_{3-24}=1\right)$, in both planning stages, and the transmission lines $n_{4-9}=1, n_{15-24}=1$ and $n_{14-23}=1$, installed in the expansion plan with security restrictions, do not appear in the expansion plan without security constraints. Further, an extra transmission line is installed in corridor 6-10 in planning with security constraints. Besides that the transmission lines $n_{11-13}=1$ and $n_{20-23}=1$ just appear in planning without security constraints.

\subsection{Colombian 93-Bus System}

The Colombian 93-bus system has 3 stages, 155 corridors, 35 generators in the first stage, 40 generators in the second stage, and 49 generators in the third stage. It has 55 load buses and a total demand of $9,750 \mathrm{MW}$ in the first stage, $12,162 \mathrm{MW}$ in the second stage and $14,559 \mathrm{MW}$ in the third stage, and a maximum number of two lines can be installed in each corridor. In Fig. 3, the base topology (solid lines) together with candidate lines (dashed lines) is shown. All the data along with the discount factor for each stage for this system can be found in LAPSEE (2012).

\subsubsection{Colombian 93-Bus Without Security Constraints}

For the case without contingency, the mathematical model has 5,519 constraints, 2,695 operation variables, and 930 binary variables. In the optimal plan, the transmission lines $n_{57-81}=2, n_{55-57}=1, n_{55-62}=1, n_{45-81}=1$ and $n_{82-85}=1$ are considered for installation in the first stage, transmission lines $n_{27-29}=1, n_{62-73}=1, n_{72-73}=1$ and $n_{19-82}=1$ for the second stage and $n_{43-88}=2, n_{15-18}=1$, 


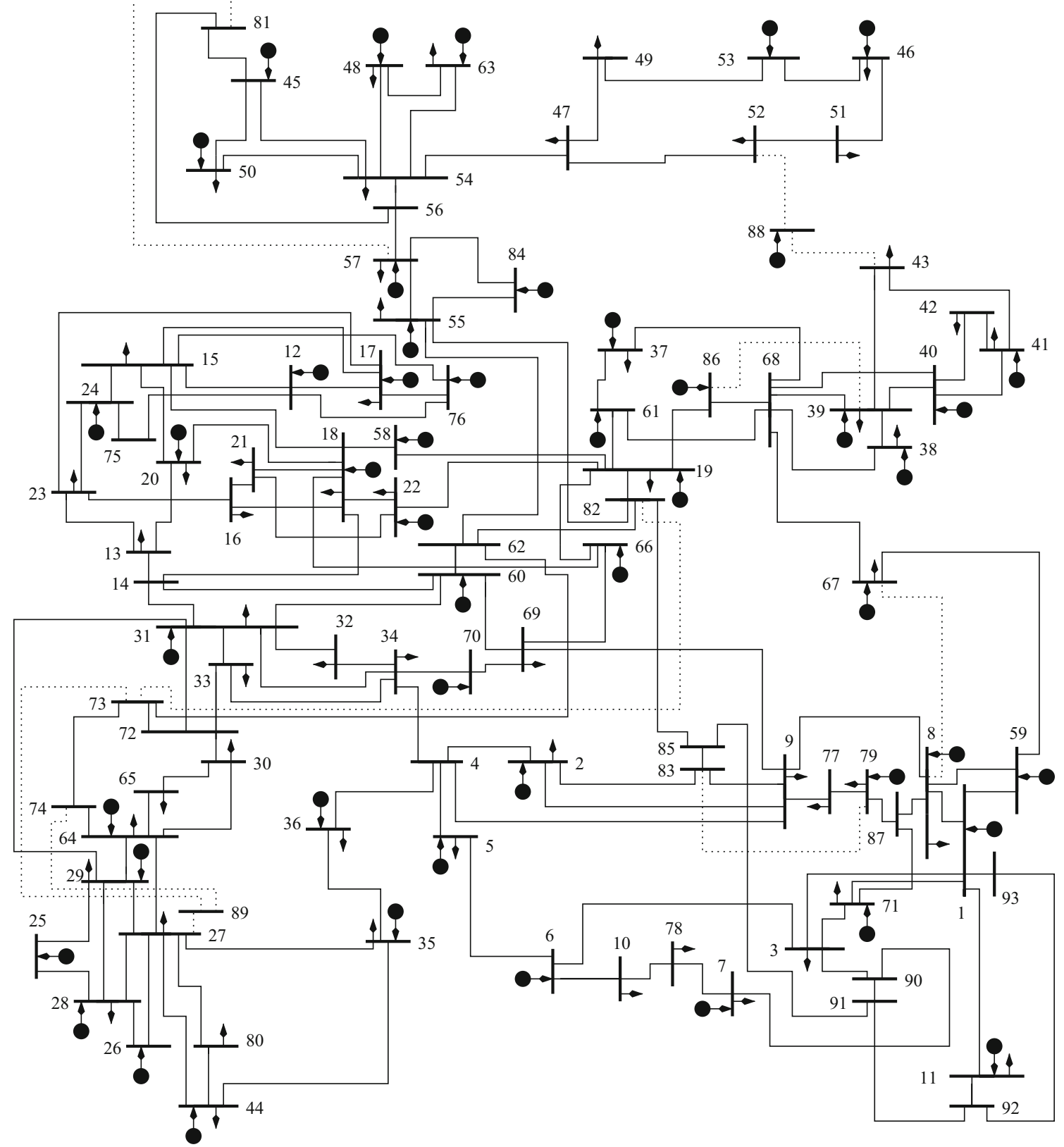

Fig. 3 The 93-bus Colombian system

$n_{30-65}=1, n_{30-72}=1, n_{55-84}=1, n_{27-64}=1$, $n_{19-82}=1$ and $n_{68-86}=1$ for the third stage. The total investment cost is US\$ 492.16, and the optimal solution is obtained in 7,599.81 s. This solution is also reported in Vinasco et al. (2011), Rahmani et al. (2013).

In this test, we do not need to use the SSRM; however, to show the efficiency of the SSRM method, the search space of the problem is reduced first, and then the MILP problem is solved. For this test the CPLEX directives are poolstub = solutions, populatelim = 5, poolgap $=0.10$, poolintensity $=4$, poolreplace $=2$, and populate $=1$. In this case, the mathematical model will have 2,125 constraints, 1,348 operation variables and 258 binary variables. The optimal solution is also obtained in this case but with much less processing time (40.07 s), which shows the efficiency of the SSRM method in reducing the computational effort of the problem.

\subsubsection{Colombian 93-Bus System with Security Constraints}

The contingency list is created using the optimum solution of the planning without contingency, that is, the existing lines and candidate lines with power flow greater than 95 and $60 \%$ of their maximum power flow are considered. Therefore, a 
contingency list containing seven transmission lines, (43-88, 45-50, 59-67, 55-62, 29-64, 48-63 and 62-73), has been created, which comprise approximately $4.51 \%$ of the total number of transmission lines. Considering the aforementioned contingency list, the mathematical model has 36,533 constraints, 15,035 operation variables, and 930 binary variables.

Due to the complexity of the mathematical problem, the solver cannot converge to the optimal solution, and the optimal solution of this test is unknown. Therefore, the SSRM is used, reduce the search space of the problem, with the same directives used as in the case without contingencies. The mathematical model has 14,314 constraints, 8 ,963 operation variables and 258 binary variables. The result shows that the transmission lines $n_{57-81}=2, n_{55-57}=1, n_{55-62}=1$ $n_{45-81}=1$ and $n_{82-85}=1$ need to be installed in the first stage, the transmission lines $n_{30-72}=1, n_{55-62}=1$, $n_{27-29}=1 n_{62-73}=2, n_{72-73}=1$ and $n_{19-82}=1$ for the second stage and $n_{52-88}=1, n_{15-18}=1, n_{30-65}=1$, $n_{55-84}=1, n_{59-67}=1, n_{27-64}=1, n_{33-72}=1$, $n_{72-73}=1, n_{68-86}=1$ for the third stage. The investment cost on lines is US\$588.08, and the processing time is $57,913.4 \mathrm{~s}$.

\subsubsection{Comparison of MTEP Planning with and without Security Constraints for Colombian 93-Bus System}

The optimal plan of the case without security constraint needs 19 new transmission lines in 16 distinct corridors; the line 19-82 is installed in the second and third stage. These lines are constructed in the three predefined stages. When considering the security constraints, the optimal plan needs 22 new transmission lines in 18 different corridors.

The difference between the investment costs in the cases with and without contingency is $19.48 \%$. Note that in both planning cases the transmission lines $\left(n_{57-81}=2, n_{55-57}=1\right.$, $n_{55-62}=1, n_{45-81}=1, n_{82-85}=1, n_{27-29}=1$, $n_{62-73}=1, n_{72-73}=1, n_{19-82}=2, n_{15-18}=1$, $n_{30-65}=1, n_{30-72}=1, n_{55-84}=1, n_{27-64}=1$ and $\left.n_{68-86}=1\right)$ are installed in 15 similar corridors. With exception of the corridor $n_{43-88}=2$, all other branches of the first case (without security constraints) are part of the second case (with security constraints).

Besides that, the corridor 19-82 has two lines in the first case and only one line in the second case. So, there are six transmission lines $\left(n_{55-62}=1, n_{62-73}=1, n_{52-88}=1\right.$, $n_{59-67}=1, n_{33-72}=1$, and $n_{72-73}=1$ ) that are different in both planning cases.

\subsection{Bolivian 57-Bus System}

The 57 bus Bolivian system has 4 stages, 92 corridors, 16 generators in the first stage, 17 generators in the second and third stage and 18 generators in the fourth stage, with 24 demand buses in the first stage, 25 demand buses in the second and third stage and 27 buses in the fourth stage. The total power demand is $962.3 \mathrm{MW}$ in the first stage, $1,029.9 \mathrm{MW}$ in the second stage, $1,229.2 \mathrm{MW}$ in the third stage and 1,733.3 MW in the fourth stage. At most, two new lines for most of the branches can be constructed and three lines for others. In Fig. 4, the base topology is shown with solid lines, as well as the candidate lines with dashed lines. All the system data and discount factors for this system can be found in LAPSEE (2012).

This system permit installation of transmission lines with 3 different characteristics, that is, a line $y$ installed in corridor $i j$, may have $k$ different characteristics showed by 1,2 , or 3. Therefore, in mathematical modelling, the installation of any line in branch $i j$ with characteristic $k$ is considered by $n_{i j, k}=1$.

\subsubsection{Bolivian 57-Bus System Without Security Constraints}

For the case without contingency, the mathematical model has 6,690 constraints, 2,700 operation variables, and 1,080 binary variables.

In the optimum solution, the transmission lines $n_{13-14,1}$ $=1, n_{36-39,1}=1$ and $n_{21-39,1}=1$ are considered for installation in the first stage, $n_{27-50,1}=1$ and $n_{21-39,1}=1$ for the second stage, $n_{43-51,1}=2$ for the third stage and $n_{24-25,1}=1, n_{41-45,1}=1, n_{52-53,1}=2, n_{43-53,1}=2$, $n_{53-51,1}=1, n_{51-54,1}=1, n_{21-55,1}=2, n_{55-20,1}=1$, $n_{55-20,2}=1, n_{55-32,1}=3$, and $n_{55-35,1}=2$ for the fourth stage with an investment cost of US\$ 71.77. The processing time for obtaining this solution is $8,708.38 \mathrm{~s}$. This solution is also reported in Vinasco et al. (2011).

Although we do not need to reduce the search space using SSRM methodology, we used that to show its performance. The following CPLEX directives are used in this case: poolstub = solutions, populatelim = 5, poolgap $=0.05$, poolintensity $=4$, poolre place $=2$ and populate $=1$. In this case, the mathematical model has 3,147 constraints, 1,412 operation variables and 444 binary variables. The optimum solution is also obtained in this test, but with much less processing time, namely $249.29 \mathrm{~s}$. Therefore, the computational effort has been reduced significantly, which once again shows the efficiency of the SSRM method.

\subsubsection{Bolivian 57-Bus System with Security Constraints}

Using the optimal plan designed for the case without contingency, the contingency list is obtained by the lines having a maximum flow greater than $90 \%$ of their maximum limit. In this case, 11 transmission lines have been chosen to 


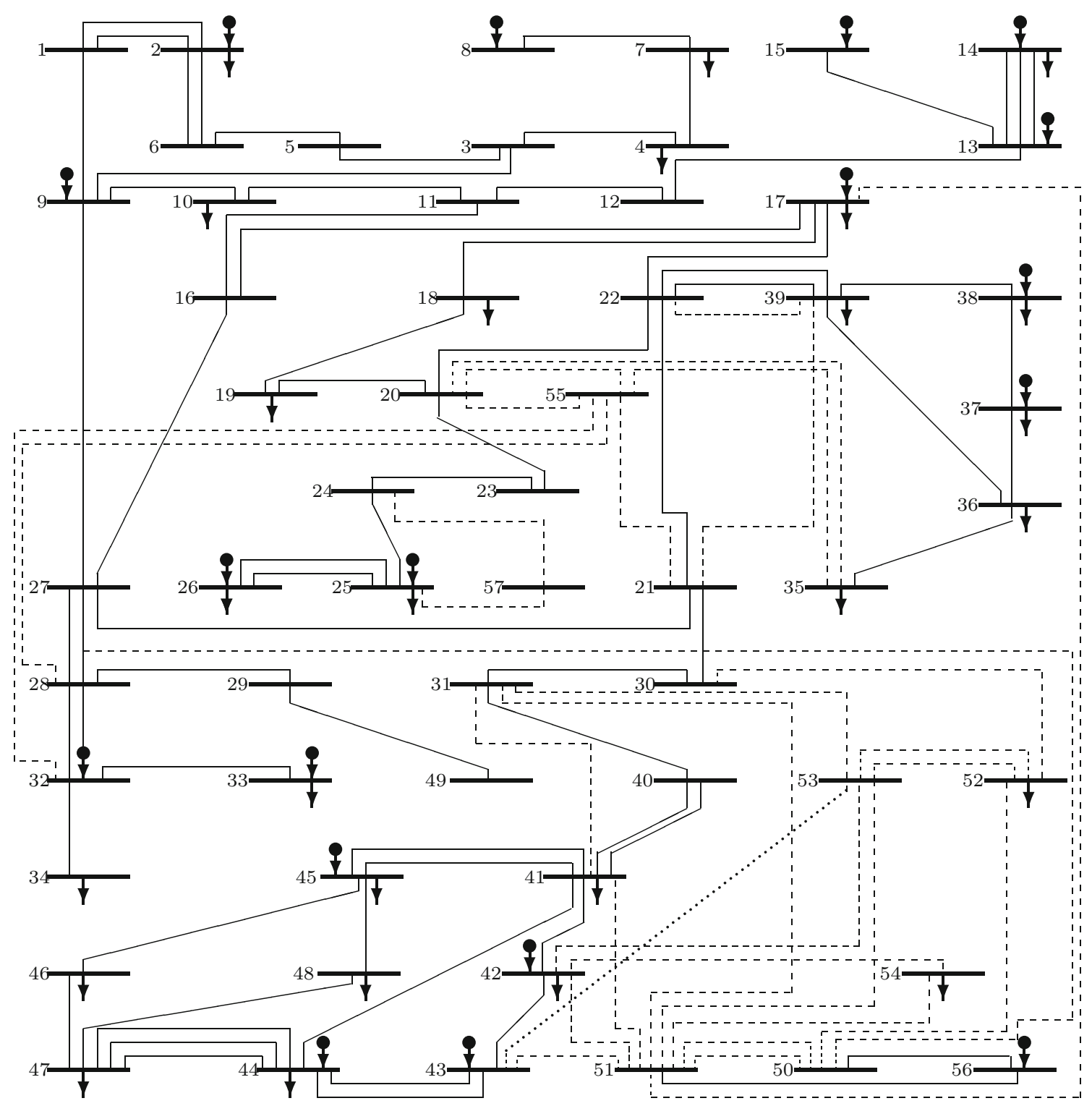

Fig. 4 The 57-bus Bolivian system

be included in the contingency list. This list contains eight transmission lines in the following corridors: 13-14, 24-25, $22-39,27-28,21-30,28-32,36-39,31-40$, and three candidate lines in the following corridors: 52-53, 53-51, and 27-5. Considering this contingency list, the mathematical model has 59,898 constraints, 20,480 operation variables, and 1,080 binary variables.

Due to the difficulty of solving the mathematical model, in the full space of the problem, the SSRM is used in this case. Therefore, the mathematical model has 29,755 constraints, 12,012 operation variables, and 444 binary variables. The proposed strategy suggested the installation of following transmission lines $n_{13-14,1}=1, n_{24-25,1}=1$, $n_{22-39,1}=1, n_{36-39,1}=2, n_{43-51,1}=1, n_{17-51,1}=$ $1, n_{55-20,2}=1, n_{55-32,1}=1$ and $n_{21-39,1}=1$ for the first stage, the transmission lines $n_{13-14,1}=1$ and $n_{17-51,1}=1$ for the second stage, and the transmission lines $n_{27-50,1}=1$ and $n_{21-39,1}=1$ for the third stage and the transmission lines $n_{41-45,1}=1, n_{42-51,1}=1$, $n_{43-51,1}=1, n_{52-53,1}=2, n_{52-53,2}=2, n_{42-53,1}=1$, $n_{43-53,1}=1, n_{53-51,1}=1, n_{51-54,1}=1, n_{21-55,1}=2$, $n_{55-20,1}=2, n_{55-32,1}=2, n_{55-35,1}=2$ for the fourth stage with an investment cost of US\$ 112.79. The proposed method finds this solution in $174,140.18 \mathrm{~s}$. In this case, there is no report studying this system, therefore, the comparison of results is not possible. However, since we used the best configuration of the CPLEX directives from previous study, which was able to obtain the optimum solution, we believe that this solution might be of a high quality. 
Table 1 Summary of the investment cost $\left(\times 10^{6}\right)$ and processing time

\begin{tabular}{|c|c|c|c|c|c|}
\hline \multirow[t]{3}{*}{ Systems } & \multicolumn{5}{|c|}{ Multistage transmission expansion planning } \\
\hline & \multicolumn{3}{|l|}{ Without contingency } & \multicolumn{2}{|l|}{ With contingency } \\
\hline & With SSRM & Without SSRM & Vinasco et al. (2011) & With SSRM & Without SSRM \\
\hline IEEE24 & $220.28 \mathrm{US} \$(<1 \mathrm{~s})$ & $220.28 \mathrm{US} \$(47.77 \mathrm{~s})$ & 220.29 US\$ (9s) & $362.64 \mathrm{US} \$(658.38 \mathrm{~s})$ & 362.64 US\$ $(14,768.30 \mathrm{~s})$ \\
\hline Colombian & $492.16 \mathrm{US} \$(40.07 \mathrm{~s})$ & 492.16 US\$ $(7,599.81 \mathrm{~s})$ & 492.17 US\$ (720s) & 588.08 US\$ $(57,913.40 \mathrm{~s})$ & Solution not obtained \\
\hline Bolivian & 71.77 US\$ $(249.29 \mathrm{~s})$ & 71.77 US\$ $(8,708.38 \mathrm{~s})$ & $71.78 \mathrm{US} \$(360 \mathrm{~s})$ & 112.79 US\$ $(174,140.18 \mathrm{~s})$ & Solution not obtained \\
\hline
\end{tabular}

\subsubsection{Comparisons Between Planning with and Without Security Constraints in Bolivian System}

The optimal plan for the case without contingency requires 24 new lines, while the planning considering security constraints required 33 new transmission lines. The difference between the investment costs in planning studies is $57.15 \%$.

The transmission lines $n_{13-14,1}=1, n_{24-25,1}=1$, $n_{36-39,1}=1, n_{21-39,1}=2, n_{27-50,1}=1, n_{41-45,1}=1$, $n_{43-51,1}=2, n_{52-53,1}=2, n_{43-53,1}=1, n_{53-51,1}=1$, $n_{51-54,1}=1, n_{21-55,1}=2, n_{55-20,1}=1 n_{55-20,2}=1$, $n_{55-32,1}=3$, and $n_{55-35,1}=2$ are common in both cases, but the model with security constraints has some other transmission lines, namely $n_{13-14,1}=1, n_{22-39,1}=1$, $n_{36-39,1}=1, n_{17-51,1}=2, n_{42-51,1}=1, n_{52-53,2}=2$, $n_{42-53,1}=1$, and $n_{55-20,1}=1$. The corridor $43-53$ is also common in both planning phases.

\subsection{Summery of Results}

Table 1 shows the processing time and investment cost of all tests studied in previous sections and subsections. Using this table, it is possible to compare the results of the test with and without $N-1$ security constraints as well as with and without using the SSRM.

Note that the processing times for the tests systems reported in Table 1, without security constraints, are less than the one reported in Vinasco et al. (2011). As shown in this table, for IEEE 24 bus system the processing time is less than a second while it is $9 \mathrm{~s}$ in Vinasco. We can observe that the solution times for Colombian and Bolivian systems using SSRM are 679 and $110 \mathrm{~s}$ less than the times reported in Vinasco. These results reveal the excellent performance of the SSRM method in reducing the processing time of the algorithm and obtaining very high-quality solutions for the TEP problems.

\section{Conclusions}

The main contribution of this paper is to present a unique mathematical model for the multistage transmission network expansion problem with $N-1$ security constraints. Unlike heuristic methods, where the security constraints are treated in separate phases, the proposed model directly considers the security constraints within the mathematical model. The advantage of the proposed model is that it has the capability of finding the optimal solution of the problem since it uses a mixed integer programming problem model.

For large-scale systems, a strategy is proposed to compute a reduced combinatorial search space for the of MTEP considering $N-1$ security constraints. This approach facilitates implementation of the proposed model and lowers the computational effort of the problems.

Comparative analysis for the model with and without security constraints is also provided. The installation of transmission lines in each planning case is studied, and a discussion of the differences in investment cost for planning cases with and without security constraints is provided. The discussion showed that the inclusion of security constraints in MTEP increases the investment cost of the expansion plan significantly; therefore, a proper definition should be made in the set of contingencies.

The results show excellent performance of the proposed approaches for solving the multistage transmission expansion planning with predefined contingency lines.

Acknowledgments This work was supported by FAPESP and CAPES.

\section{References}

Asadamongkol, S., \& Eua-arporn, B. (2009). Transmission system expansion planning with consideration of $n-1$ security constraint. In International conference on electrical engineering/electronics, computer, telecommunications and information technology, Pattaya, Chonburi (Vol. 1, pp. 218-221).

CEIDS. (2012). Transmission operations-Contingency analysis baseline function.http://www.intelligrid.info/IntelliGrid_Architec ture/Use_Cases/TO_Contingency_Analysis_Baseline.htm.

CPLEX. (2010). Optimization subroutine library guide and reference, version 12.2. Incline Village, NV: CPLEX Division, ILOG Inc.

da Silva, E. F. (2013). Planejamento Estocástico da Expansão da Rede de Transmissão de Energia Elétrica Multiestágio Considerando Restrições de Segurança (Stochastic programming for the Multistage Transmission Expansion Planning Considering Security 
Constraints). Doutorado em engenharia elétrica. Universidade Estadual Paulista. Faculdade de Engenharia de Ilha Solteira, Ilha Solteira, SP.

da Silva, E. F., Resende, A. S., \& Rider, M. J. (2013). Planejamento da expansão da rede de transmissão multiestágio com cenários e restrições de segurança (multistage transmission expansion planning with scenarios and security constraints), $45^{\circ}$ simpósio brasileiro de pesquisa operacional. Natal, Rio Grande do Norte, Brazil.

Fourer, R., Gay, D. M., \& Kernighan, B. W. (2003). AMPL: A modeling language for mathematical programming. Pacific Grove, CA: Brooks/Cole-Thomson Learning.

Garver, L. L. (1970). Transmission network estimation using linear programming. IEEE Transactions on Power Apparatus and Systems, 89(7), 1688-1697.

Haffner, S., Monticelli, A., Garcia, A. V., \& Romero, R. (2001). Specialized branch and bound algorithm for transmission network expansion planning. IEE Proceedings of Generation, Transmission and Distribution, 148(5), 482-488.

LAPSEE. (2012). Transmission expansion planning test systems. www.feis.unesp.br/\#!/departamentos/engenharia-eletrica/pesqui sas-e-projetos/lapsee/downloads/.

Lu, M., Dong, Z. Y., \& Saha, T. K. (2005). A framework for transmission planning in a competitive electricity market. In IEEE/PES transmission and distribution conference and exposition: Asia and Pacific (p. 16).

Maghouli, P., Hosseini, S. H., Buygi, M. O., \& Shahidehpour, M. (2009). A multi-objective framework for transmission expansion planning in deregulated environments. IEEE Transactions on Power Systems, 24(2), 1051-1061.

Monticelli, A., Santos, A, Jr, Pereira, M. V. F., Cunha, S. H., Parker, B. J., \& Praça, J. C. G. (1982). Interactive transmission network planning using a least-effort criterion. IEEE Transactions on Power Apparatus and Systems, PAS-101(10), 3919-3925.

Oliveira, G. C., S., Pereira, M. V. F., \& Thomé, L. M. (2004). Multi-stage transmission expansion planning considering multiple dispatches and contingency criterion, Anais do XV Congresso Brasileiro de Automática (CBA 2004) (pp. 1-5). Universidade Federal do Rio Grande do Sul, Gramado, Brazil.
Rahmani, M., Romero, R., \& Rider, M. (2013). Strategies to reduce the number of variables and the combinatorial search space of the multistage transmission expansion planning problem. IEEE Transactions on Power Systems, 28(3), 2164-2173.

Romero, R., Monticelli, A., Garcia, A., \& Haffner, S. (2002). Test systems and mathematical models for transmission network expansion planning. IEE Proceedings of Generation, Transmission and Distribution, 149(1), 27-36.

Samarakoon, H., Shrestha, R., \& Fujiwara, O. (2001). A mixed integer linear programming model for transmission expansion planning with generation location selection. Electrical Power and Energy Systems, 23(4), 285-293.

Seifu, A., Salon, S., \& List, G. (1989). Optimization of transmission line planning including security constraints. IEEE Transactions on Power Systems, 4(4), 1507-1513.

Silva, I. J., Rider, M. J., Romero, R., Garcia, A. V., \& Murari, C. A. (2005). Transmission network expansion planning with security constraints. IEE Proceedings of Generation, Transmission and Distribution, 152(6), 828-836.

Verma, A., Bijwe, P. R., \& Panigrahi, B. K. (2009). Heuristic method for transmission network expansion planning with security constraints and uncertainty in load specifications. In Transmission and distribution conference and exposition: Asia and Pacific, Seoul (pp. 1-4).

Verma, A., Bijwe, P. R., \& Panigrahi, B. K. (2010). Harmony search algorithm for transmission network expansion planning. Generation, Transmission \& Distribution, IET, 4(6), 663-673.

Vinasco, G., Rider, M., \& Romero, R. (2011). A strategy to solve the multistage transmission expansion planning problem. IEEE Transactions on Power Systems, 26(4), 2574-2576.

Wood, A. J., Wollenberg, B. F., \& Sheblé, G. B. (2013). Power generation, operation and control (3rd ed.). New York: Wiley.

Xu, Z., Dong, Z. Y., \& Wong, K. P. (2006). A hybrid planning method for transmission networks in a deregulated environment. IEEE Transactions on Power Systems, 21(2), 925-932.

Xu, Z., Dong, Z. Y., Wong, K. P., \& Fan, Z. (2009). Multi-objective transmission planning. In Power and energy engineering conference: Asia and Pacific, Wuhan (pp. 1-4). 